\title{
Evaluation of the mood repair hypothesis of compulsive buying
}

\author{
Alishia D. Williams \\ Clinical Research Unit for Anxiety and Depression, University of New South Wales at St. Vincent's Hospital, Sydney, Australia \\ Email: alishia.williams@unsw.edu.au
}

Received 1 January 2012; revised 18 February 2012; accepted 5 March 2012

\begin{abstract}
Compulsive buying (CB) is a proposed disorder of dysregulated buying behaviour that is associated with high rates of Axis I comorbidity, particularly depression and anxiety. It has been proposed that purchasing behaviours may serve as a maladaptive means of alleviating negative affect in vulnerable individuals. The aim of the current study was to experimentally manipulate affect to test this mood repair hypothesis. Compulsive buyers $(n=26)$ and pathological gamblers $(n=23)$ diagnosed using structured clinical interviews (SCID) and healthy controls $(n=24)$ were randomly assigned to either a negative or positive mood-induction procedure (MIP) and participated in an experimental buying task. Results revealed that, irrespective of mood induction condition, compulsive buyers reported a greater urge to acquire items, purchased more items, and spent a greater total amount of money during the buying task when compared to the healthy control group. Compulsive buyers were also faster than pathological gamblers in making decisions to purchase, even after controlling for motor impulsivity (BIS). There was, however, no main effect of mood-induction condition or group by condition interaction. Limitations and future directions are discussed.
\end{abstract}

Keywords: Compulsive Buying; Acquisition; Mood Repair; Depression

\section{INTRODUCTION}

In the research planning agenda leading up to DSM-V (Diagnostic and Statistical Manual of Mental Disorders; [1]), there has been a call for further research into the phenomenology of compulsive buying (CB). CB is defined as chronic, repetitive purchasing behaviour that does not occur in the context of mania, is difficult to control, and is associated with frequent buying of more than can be afforded, of items that are not needed, or shopping for longer periods of time than intended [2]. Estimated prevalence data collected in the United States indicates approximately $5.8 \%$ of males and females report clinically significant $\mathrm{CB}$ behaviours while a recently published study in Germany reported an estimated point prevalence of $6.9 \%$ across the genders [3]. Converging data suggests that compulsive buying (CB) represents a significant concern from a human disease perspective, resulting in marked distress, marital and social conflict, significant financial debt [4-6] and impacting upon general quality of life and work functioning [7]. To date, long-term follow-up studies of CB are absent, but research suggest that the course is chronic or recurrent $[8$, 9] highlighting the severity of the condition. In terms of purchasing behaviour, the most common items bought are clothing, shoes, compact discs, jewellery, cosmetics, and various household items [8-10]. During a buying episode it has been estimated that average spending ranges between $\$ 92$ - $\$ 110$ US dollars [8,9]. There is a high prevalence rate of Axis I disorders in CB compared to that reported in epidemiological samples [11], particularly depression and anxiety $[2,5]$ and $\mathrm{CB}$ is documented to occur in response to depressive states $[10,12]$. Indeed, in a study of the antecedents and consequences of buying episodes negative emotional sates, including depression, were reported as the most common antecedent prompting a buying episode, followed by subsequent positive emotions or a sense of relief [10]. It has therefore been proposed that purchasing behaviours may serve as a maladaptive means of alleviating negative affective states in vulnerable individuals [12], particularly in those who demonstrate deficits in emotion-regulation [13]. Faber and Christenson [12] reported that the majority of their sample of compulsive buyers $(95.8 \%)$ reported a shift in mood following a purchase compared to only $29 \%$ of their comparison sample. For the compulsive buyers, the shift in mood was predominantly from a negative to a more positive mood state, supporting the proposal that purchasing behaviours may serve a mood-repair function. Faber and Christenson [12] further theorized that compulsive buyers may become reli- 
ant on buying behaviours as a means of alleviating negative affect through a process of negative reinforcement. Other researchers in the field have also proposed a mood-repair function [14]. The aims of the current study were to experimentally manipulate affect to test the prediction that compulsive buyers regulate negative mood through purchasing behaviour. It was hypothesized that compulsive buyers would report a greater urge to obtain items, and subsequently purchase more items, displayed during a computer-based buying task compared to a healthy control and clinical comparison group. It was further predicted that compulsive buyers assigned to the negative mood induction condition would report the greatest urge and spend the most total amount of money relative to all other groups. Finally, it was predicted that compulsive buyers in the negative mood induction condition would demonstrate a facilitative mood repair effect relative to all other groups evidenced by a greater increase in positive affect following the buying task.

\section{METHODS}

\subsection{Participants}

\subsubsection{Pathological Gambling Group}

This group included 14 males and 9 females with a mean age of $39.43(S D=11.83)$ who met DSM-IV-TR criteria for pathological gambling based on a structured clinical interview. South Oaks Gambling Screen (SOGS) scores ranged from 8 to 20 with a mean of $15.30(S D=4.03)$. Current and lifetime diagnostic information obtained from the full Structured Clinical Interview for DSM Disorders (SCID-I/NP Non-patient Edition; [15]) is presented in Table 1.

\subsubsection{Compulsive Buying Group}

This group included 24 females and 2 males with a mean age of $28.31(S D=11.47)$ who all met proposed DSM criteria [2] and SCID Impulse Control Disorder criteria for compulsive buying. Current and lifetime diagnostic information is presented in Table 1.

\subsubsection{Healthy Control Group}

Twenty-five individuals who did not endorse any current or lifetime mood, anxiety, substance-abuse, head injury, psychotic disorder, or gambling pathology were initially included in this group. One participants who scored above the cut-score of 5 of the SOGS was excluded resulting in a final sample of 19 females and 5 males with a mean age of $28.88(S D=5.84)$.

\section{MEASURES}

1) The Positive and Negative Affect Scales (PANAS; [16]). The PANAS consists of two 10-item scales to
Table 1. Current and lifetime Axis I diagnoses for the pathological gambling group and the compulsive buying group.

\begin{tabular}{ccccc}
\hline & \multicolumn{2}{c}{ Pathological Gamblers } & \multicolumn{2}{c}{ Compulsive Buyers } \\
\cline { 2 - 5 } Diagnosis & Current & Lifetime & Current & Lifetime \\
\cline { 2 - 5 } & $n(\%)$ & $n(\%)$ & $n(\%)$ & $n(\%)$ \\
\hline $\begin{array}{c}\text { Mood } \\
\text { Disorder } \\
\text { Anxiety }\end{array}$ & $5(22 \%)$ & $10(43 \%)$ & $6(23 \%)$ & $13(50 \%)$ \\
$\begin{array}{c}\text { Disorder } \\
\text { Other }\end{array}$ & $5(22 \%)$ & $9(39 \%)$ & $14(54 \%)$ & $2(8 \%)$ \\
$\begin{array}{c}\text { Skin Picking } \\
\text { Intermittent }\end{array}$ & $0(0)$ & $0(0)$ & $1(3 \%)$ & $1(3 \%)$ \\
$\begin{array}{c}\text { Explosive } \\
\text { Alcohol } \\
\text { Abuse/ }\end{array}$ & $0(0)$ & $5(22 \%)$ & $0(0)$ & $0(0)$ \\
$\begin{array}{c}\text { Dependency } \\
\begin{array}{c}\text { Substance } \\
\text { Abuse/ }\end{array}\end{array}$ & $0(0)$ & $2(8 \%)$ & $0(0)$ & $0(0)$ \\
$\begin{array}{c}\text { Dependency } \\
\text { Bulimia } \\
\text { Nervosa }\end{array}$ & $0(0)$ & $0(0)$ & $1(3 \%)$ & $0(0)$ \\
\hline
\end{tabular}

*Percentages do not sum to 100 due to multiple comorbidities.

measure positive and negative affect. Both the PANAS-P and the PANAS-N consist of 10 items with possible scores from 10 to 50 . Increased scores reflect higher positive or negative affect, respectively. The PANAS demonstrates good psychometric properties [16].

2) Barratt Impulsiveness Scale (BIS-II; [17]). The BIS-II is a widely-used 30-item self-report questionnaire of impulsivity with good psychometric properties [18]. The BIS Motor subscale was used to control for motor impulsivity when indexing response latencies during the buying task.

3) Depression Anxiety Stress Scales (DASS; [19]). The DASS is a validated and widely-used 21-item self-report measure of depression, anxiety and stress symptoms with good reliability and internal consistency [20].

4) The Yale Brown Obsessive Compulsive ScaleShopping Version (YBOCS-SV; [21]). The YBOCS-SV is a 10 -item measure of preoccupations, behaviours, and subsequent distress associated with compulsive buying. It has shown promise as an index of compulsive buying severity that is sensitive to clinical change [21]. Cronbach's alpha was 0.92 in the current sample.

5) The South Oaks Gambling Screen (SOGS; [22]). The SOGS is the most widely used instrument for assessing problem gambling (e.g. National Research Council). Scores range from 0 to 20 , with scores $\geq 5$ indicative of probable pathological gambling [22] (Lesieur and 
Blume, 1987). Typically, a moderate to high correlation exists between SOGS scores, DSM criteria, and other indices of gambling frequency and severity [23,24].

6) Structured Clinical Interview for DSM Disorders (SCID-I/NP Non-patient Edition; [14]). The SCID is a semi-structured interview for making the major DSM-IV Axis I diagnoses. The SCID was used to determine current and lifetime history in the clinical groups.

7) Structured Clinical Interview for DSM-IV-TR (SCID) for impulse-control disorders not elsewhere classified (SCID-ICD; [25]). The SCID-ICD contains 6 modules for disorders of impulse-control currently in the DSMIV-TR (intermittent explosive disorder, kleptomania, pyromania, trichotillomania, ICD-NOS, and pathological gambling) as well as four modules not currently defined in the DSM (impulsive-compulsive buying, impulsivecompulsive non-paraphilic sexual behaviour, impulsivecompulsive internet use, and impulsive-compulsive skin picking).

8) Structured Clinical Interview for DSM DisordersScreening Module (SCID-I/NP; [14]). The SCID screening module was used to screen participants recruited from the community and to rule out any history of Axis I symptoms in the healthy control group.

9) Urge Ratings. This scale was created for the purposes of the present study. Respondents rated their current urge to have the item displayed during the buying task on a scale of $0=$ No Urge to $4=$ Extreme Urge.

\section{PROCEDURE}

Ethical approval was obtained by the Human Research Ethics Committee of St. Vincent's Hospital and the University of New South Wales. Participants were recruited via separate advertisements placed in the community either targeting individuals with no current or lifetime history of mental health concerns to participate in a study of buying behaviours, or through advertisements placed on mental health websites and in a local gambling treatment unit seeking individuals with symptoms consistent with compulsive buying or pathological gambling. All participants were initially screened over telephone by a registered psychologist using the screening module of the SCID-I/NP, and those who endorsed symptoms consistent with $\mathrm{CB}$ or $\mathrm{PG}$ also were screened using the relevant module of the SCID-ICD. Current substance abuse, history of head injury, and psychotic disorder were exclusion criteria for all participants. Eligible respondents were then invited to attend a research session where they completed the battery of self-report questionnaires, full SCID (clinical groups only), and participated in the mood induction and shopping task describe below. Informed consent was obtained for each participant prior to commencement of the study and a small financial reim- bursement was provided in exchange for participants' time. The template is used to format your paper and style the text. All margins, column widths, line spaces, and text fonts are prescribed; please do not alter them. You may note peculiarities. For example, the head margin in this template measures proportionately more than is customary. This measurement and others are deliberate, using specifications that anticipate your paper as one part of the entire journals, and not as an independent document. Please do not revise any of the current designations.

\subsection{Mood Induction}

A tri-fold mood induction procedure was used that included the Velten MIP [26] and accompanying music induction (Prokofiev's "Russia Under the Mongolian Yolk" played at half speed for the negative condition and Vivaldi's "Spring Concerto" for the positive condition) followed by an incubation period. The Velten MIP was chosen based on evidence that it is among the most effective MIPs [27]. Fifty-six of the original 60 Velten statements were used with the wording modified for 3 items as follows: reference to study was replaced by "work", reference to parents was changed to family, the word "GOD" in the positive induction statement "GOD, I feel great" was replaced with the word "WOW". Velten statements were displayed to participants on a laptop computer using Powerpoint. The music played simultaneously through Philips Noise Reduction headphones for 8 minutes. The Velten statements were matched in duration to the music followed by an incubation period wherein participants read instructions designed to maintain their focus on either the positive or negative mood. The incubation period was included based on research that has demonstrated that a short incubation period can intensify the experience of affective states [28] and to ensure that the mood induction effects endured beyond the manipulation. Incubation instructions were taken from Sinclair et al. [28].

\subsection{Buying Task}

The buying task required participants to rate their current urge to obtain each of the 40 items displayed individually on a computer screen and to make a decision whether or not to "purchase" the displayed item at the given fixed price. Participants were therefore displayed each item twice; once to obtain the urge rating and a subsequent time to record the decision to purchase (YES/NO) and to record latency to respond. All stimuli were presented using Medialab software with images standardized in size of $300 \times 300 \mathrm{px}$ and presented at a fixed position on the screen. All items were free of branding information and logos to reduce the likelihood of brand preference 
impacting upon purchase decisions. Items were priced based on the valuation of comparable items available in Australia. Individual items ranged in cost from $\$ 2.00$ $\$ 130.00$ dollars and the total amount purchasable was $\$ 1600.00$ dollars. The order of presentation was randomized across participants. Response latencies for the decision to purchase were recorded in milliseconds for each item. Two versions of the shopping task were created that contained unisex and gender specific items, therefore males and females were displayed items that were selected on their relevance to $\mathrm{CB}$ as demonstrated in previous research $[8,9]$. Each participant completed 3 practice items (food items) to familiarize themselves with the task. Instructions were as follows: In this section you will be helping the researchers develop a future study. You will be presented with various items and asked to rate your urge or desire to have each item and then given the opportunity to "purchase" each item. You will not actually spend any money during this task, but we ask you to imagine that you are actually spending your own money by making the decision to purchase. Remember that your responses will be very helpful in developing a future study so please respond honestly.

\section{RESULTS}

\subsection{Sample Characteristics}

Groups were matched with respect to education, $\chi^{2}(8)=$ 14.74, $p>0.05$, ethnicity, $\chi^{2}(8)=5.86, p>0.05$, and marital status, $\chi^{2}(8)=8.29, p>0.05$. Average annual income did not differ with salaries of $\$ 33269.43(S D=$ 22702.49), \$34684.62 ( $S D=28822.16)$, and $\$ 40652.17$ $(S D=25055.29)$ for the $\mathrm{HC}, \mathrm{CB}$, and PG groups, respectively, $F(2,72)=0.58, p>0.05$. There was a gender imbalance, $\chi^{2}(2)=16.49, p<0.001$ with more females in the CB group. This difference is not unexpected given the greater proportion of females $(80 \%-95 \%)$ who volunteer for CB research [29]. There was also an imbalance in age $F(2,72)=8.50, p<0.001$, with participants in the PG group significantly older than in the $\mathrm{CB}$ and $\mathrm{HC}$ group, $p<0.001$. Age did not, however, correlate with any of the outcome variables, all $p$ 's $>0.05$. On the measures of gambling and buying pathology it was expected that the two clinical groups would score differenttially on these measures. For the SOGS there was a main effect of group, $F(2,70)=172.51, p<0.001$. As expected the PG group scored significantly higher than both the $\mathrm{CB}$ and $\mathrm{HC}$ groups, $p$ 's $<0.001$, whose scores did not differ, $p>0.05$. For the YBOCS-SV there was a main effect of group, $F(2,70)=15.23, p<0.001$. As expected the $\mathrm{CB}$ group scored significantly higher than both the PG and HC groups, $p$ 's $<0.001$, whose scores did not differ, $p=0.05$. For general psychopathology there was a main effect of group, $F(3,70)=54.19, p<$ 0.001 , with the $\mathrm{HC}$ group scoring significantly lower on all subscales of the DASS in comparison to the CB and PG group, $p$ 's $<0.001$. The CB and PG group did not differ on any subscales, $p>0.05$. For BIS Motor Impulsivity there was a main effect of group, $F(2,61)=8.05, p$ $=0.001$, with the $\mathrm{HC}$ group scoring significantly lower than the CB and PG groups, $p$ 's $<0.01$, who did not differ from one another, $p>0.05$. Means and standard deviations are reported in Table 2.

\subsection{Effectiveness of Mood Induction}

To ensure that the mood induction procedure was effective in inducing positive and negative affect, separate mixed models ANOVAs with time as a repeated factor and condition as the between-subjects factor were conducted for PANAS Positive and PANAS Negative. Eta squared $\left(\eta^{2}\right)$ values were calculated to report effect sizes. For PANAS Positive ANOVA revealed a main effect of

Table 2. Planned comparisons of groups on self-report measures.

\begin{tabular}{ccccc}
\hline \multirow{2}{*}{ Measure } & Pathological Gamblers & Compulsive Buyers & Healthy Controls & \multirow{2}{*}{$F$} \\
\cline { 2 - 4 } & $M(S D)$ & $M(S D)$ & $M(S D)$ & $172.51^{* * *}$ \\
SOGS & $15.30(4.03)^{\mathrm{a}}$ & $1.53(3.07)^{\mathrm{b}}$ & $1.00(1.21)^{\mathrm{b}}$ & $15.23^{* * *}$ \\
YBOCS-SV & $8.78(7.51)^{\mathrm{a}}$ & $14.69(5.25)^{\mathrm{b}}$ & $5.20(5.57)^{\mathrm{a}}$ & $8.05^{* * *}$ \\
Motor Impulsivity & $28.08(4.32)^{\mathrm{a}}$ & $28.04(5.46)^{\mathrm{a}}$ & $23.05(3.07)^{\mathrm{b}}$ & $9.86^{* * *}$ \\
DASS TOTAL & $38.00(23.57)^{\mathrm{a}}$ & $37.23(26.82)^{\mathrm{a}}$ & $13.16(13.02)^{\mathrm{b}}$ & $6.48^{* *}$ \\
DASS Depression & $12.95(10.33)^{\mathrm{a}}$ & $11.92(11.65)^{\mathrm{a}}$ & $3.91(5.08)^{\mathrm{b}}$ & $7.95^{* *}$ \\
DASS Anxiety & $9.91(7.87)^{\mathrm{a}}$ & $10.23(8.41)^{\mathrm{a}}$ & $2.91(4.71)^{\mathrm{b}}$ & $8.33(5.39)^{\mathrm{b}}$ \\
DASS Stress & $15.13(9.08)^{\mathrm{a}}$ & $15.07(9.73)^{\mathrm{a}}$ & $6.93^{* * *}$ \\
\hline
\end{tabular}

Note: SOGS = South Oaks Gambling Screen; YBOCS-SV = The Yale Brown Obsessive Compulsive Scale-Shopping Version; Motor Impulsivity = Barratt Impulsiveness Scale-Motor Impulsivity Subscale; DASS = Depression Anxiety Stress Scale. Means with different superscripts are significantly different. 
time, $F(1,69)=7.64, p<0.01, \eta^{2}=0.10$, and a significant time $\mathrm{x}$ condition interaction, $F(1,69)=51.21, p<$ $0.001, \eta^{2}=0.43$. Importantly there was no main effect of group or group $\mathrm{x}$ condition interaction, $p$ 's $>0.05$. The significant time $\mathrm{x}$ condition interaction was decomposed by conducting paired-samples t-tests separately in each condition. For the positive condition, mean PANAS positive scores increased significantly from baseline $(M=$ $26.60, S D=8.13)$ to the post mood induction phase $(M=$ $30.63, S D=9.66), t(40)=-4.05, p<0.001$. For the negative condition, mean PANAS positive scores decreased significantly from baseline $(M=28.91, S D=9.31)$ to the post mood induction phase $(M=19.91, S D=8.56), t(33)$ $=5.73, p<0.001$. For PANAS Negative, ANOVA revealed a main effect of time, $F(2,69)=5.62, p<0.05, \eta^{2}$ $=0.08$, and a significant time $\mathrm{x}$ condition interaction, $F(2$, $69)=28.57, p<0.001, \eta^{2}=0.23$. For the positive condition, mean PANAS Negative scores decreased significantly from baseline $(M=15.34, S D=5.97)$ to the post mood induction phase $(M=13.46, S D=5.31), t(40)=$ $3.11, p<0.01$. For the negative condition, mean PANAS Negative scores increased significantly from baseline $(M$ $=14.41, S D=4.91)$ to the post mood induction phase $(M$ $=19.55, S D=6.94), t(33)=-4.14, p<0.001$. Thus the mood induction procedure was successful in inducing both positive and negative affect in the respective conditions.

\subsection{Urge to Purchase}

For average purchase urge there was a main effect of group, $F(2,66)=11.38, p<0.001, \eta^{2}=0.24$. There was no main effect of condition, or group $\mathrm{x}$ condition interaction, $p$ 's $>0.05$. Planned comparisons revealed that the CB group reported the greatest urge to purchase $(M=$ 2.63, $S D=0.73)$, compared to the $\mathrm{HC}(M=1.76, S D=$ $0.43)$ and PG groups $(M=2.18, S D=0.71), p$ 's $<0.05$. Urge rating were significantly higher in the $\mathrm{PG}$ group compared to the HC group, $p<0.05$.

\subsection{Total Money Spent and Items Purchased}

To compare the total amount of money spent during the buying task across groups, an ANCOVA was conducted with average annual income included as the covariate. Prior to the main analysis, the homogeneity of the regression assumption for the covariate was tested. Importantly, there was no interaction between condition and the covariate, $F(1,62)=0.19, p>0.05$. ANCOVA revealed a main effect of group, $F(2,65)=10.37, p<0.01, \eta^{2}=$ 0.22 . There was no main effect of condition or group $\mathrm{x}$ condition interaction, $p$ 's $>0.05$. Planned comparisons revealed that the $\mathrm{HC}$ group spent significantly less money $(M=\$ 109.00, S D=124.46)$ than the PG $(M=$ $\$ 481.60, S D=443.19)$ and the $\mathrm{CB}(\mathrm{M}=\$ 489.29, S D=$
315.43) groups, $p$ 's $<0.001$. The amount spent by the CB and PG group did not differ, $p>0.05$. A similar pattern emerged when absolute number of items purchased was considered, $F(2,69)=10.58, p<0.001, \eta^{2}=0.22$, with the HC group purchasing significantly fewer items $(M=$ $4.56, S D=4.75)$ than the $\mathrm{CB}$ group $(M=11.91, S D=$ $5.20)$ and the PG group $(M=12.65, S D=9.58), p$ 's < 0.001 . The $\mathrm{CB}$ and $\mathrm{PG}$ groups did not differ in the number of items purchased, $p>0.05$.

\subsection{Effect of Gender on Total Money Spent and Items Purchased}

To test for any differential responses between males and females gender was included as a between-groups factor with group in separate ANCOVAs with total amount of money spent and total number of items purchased entered as dependent variables and income entered as the covariate. For total amount of money spent there were significant main effects of group and gender, $p$ 's $<0.05$, qualified by a significant group $\mathrm{x}$ gender interaction, $F(2$, $64)=3.58, p<0.05, \eta^{2}=0.07$. Comparisons revealed that there were no gender differences in the HC group or the $\mathrm{CB}, p$ 's $>0.05$. There was a gender difference for the total amount of money spent in the PG group, $t(21)=$ $3.44, p<0.01$, with males spending significantly more money $(M=590.42, S D=402.41)$ than females $(M=$ $164.22, S D=184.31)$. For total number of items purchased there was a main effect of group $F(2,65)=5.73$, $p<0.01, \eta^{2}=0.12$. There was no main effect of gender or group $\mathrm{x}$ gender interaction, $p$ 's $>0.05$.

\subsection{Mood Repair}

To compare change in affect ratings following the buying task, mean PANAS scores following the buying task were subtracted from mean PANAS scores following the mood induction. For PANAS Positive mood change there was a main effect of condition, $F(1,64)=8.10, p<0.01$, $\eta^{2}=0.11$, indicating recovery from the mood induction was greater in the positive condition, but there was no main effect of group or condition $\mathrm{x}$ group interaction, $p$ 's $>0.05$. For PANAS Negative there was a main effect of condition, $F(1,64)=23.08, p<0.001$, indicating recovery from the mood induction was greater in the negative condition, $\eta^{2}=0.26$, but there was no main effect of group or condition $\mathrm{x}$ group interaction, $p$ 's $>0.05$.

\subsection{Comparisons between the $C B$ and $P G$ Groups}

\subsubsection{Motor Impulsivity and Reaction Time}

Given that impulsivity is a proposed defining feature of both $\mathrm{CB}$ and $\mathrm{PG}$, response-latencies to items during the buying task were compared between the two clinical 
groups only. After selecting out the HC group, ANCOVA controlling for motor impulsivity (BIS Motor subscale) was conducted. There was a main effect of group, $F(1$, $41)=4.10, p<0.05, \eta^{2}=0.08$. There was no main effect of condition or condition $\mathrm{x}$ group interaction, $p$ 's $>0.05$. The CB group was significantly faster at making decisions to purchase $(M=1048.99, S D=256.90)$ than the PG group $(M=1260.92, S D=424.74)$ despite comparable levels of behavioural (motor) impulsivity.

\subsubsection{Effect of Depression}

To investigate whether depression history (SCID current/ lifetime) resulted in a differential response to the mood induction and decisions to purchase in the $\mathrm{CB}$ and $\mathrm{PG}$ groups, ANCOVA with depression status added as a between-groups factor and income as the covariate was conducted. There was no main effect of group, condition, or depression status, $p$ 's $>0.05$, but there was a significant depression status $\mathrm{x}$ condition interaction, $F(1,38)=$ $9.27, p<0.01, \eta^{2}=0.16$. No other effects were significant, $p>0.05$. Comparisons revealed that irrespective of group, participants in the negative condition without a history of depression spent significantly more money $(M$ $=\$ 649.30, S D=374.08)$ than participants with a depression history $(M=\$ 189.08, S D=135.67), F(1,20)=$ $15.80, p<0.001$. The total amount of money spent did not vary as a function of depression history in the positive condition, $p>0.05$.

\section{DISCUSSION}

The current study experimentally manipulated affect using a tri-fold mood induction procedure to test the prediction that compulsive buyers regulate negative affective states through purchasing behaviour. Specifically, it was predicted that following a negative mood induction, compulsive buyers would report a greater urge to purchase merchandise and subsequently spend more money during an experimental buying task relative to all other groups. The first hypothesis was supported. Irrespective of mood induction condition, compulsive buyers reported a greater urge to acquire items during the buying task, purchased more items, and spent a greater total amount of money during the task when compared to a healthy control group. The inclusion of a group of pathological gamblers also allowed a clinical comparison on measures of impulsive responding to help clarify the nature of the relationship between affect, impulsivity, and buying behaviours. Consistent with the literature that argues for a relationship between $\mathrm{CB}$ and PG [30], pathological gamblers behaved similarly to compulsive buyers during the buying task in terms of items bought and total amount of money spent. The $\mathrm{CB}$ group was faster than the $\mathrm{PG}$ group in making decisions to purchase, even after con- trolling for motor impulsivity. Although this difference was statistically significant, the mean difference in reaction times may not be that clinically relevant, except when applied to online buying behaviours when purchase transactions typically only rely on a series of mouse clicks.

Interestingly there were no general gender differences on the buying task, with the exception that male gamblers spent significantly more money during the task than female gamblers. This finding may simply be related to the fact that money plays a central role in pathological gambling. Given the imbalance in gender across the two clinical groups this finding should be interpreted with caution. Future research would benefit from a more representative sample of both genders.

The primary hypothesis, that buying behaviours would serve a mood repair function, was not supported. There was no condition by group interaction for changes in either positive or negative affect following the buying task. Therefore, there was no evidence of differential mood repair effect in the $\mathrm{CB}$ group despite the effectiveness of the mood induction procedure. Although descriptive studies provide a convincing rationale in support of the mood repair hypothesis, the current study represents the first published attempt to test this proposal experimentally. Although participants were encouraged to respond as if they were making the decision to purchase using their own money, the nature of the task was necessarily artificial. It may be the case that under natural buying conditions positive affect is induced through actual purchase behaviour that leads to a sense of gratification via ownership of the item bought. This would seem unlikely however based on research that reports a genuine lack of interest in, or use of, purchased items [31]. Although the mood induction procedure resulted in significant changes in state mood, the level of induced negative affect was moderate. Current conceptualizations do not specify whether a threshold of negative affect/ dysphoria exists that is necessary to result in a pathological buying episode, but Faber and Vohs [32] recently proposed that extremely high levels of negative affect are required. Experimental investigations of this proposal may not be feasible due to ethical reasons. Other necessary aspects of the buying episode have also recently been proposed; namely the level of engagement in the buying episode or the extent to which an individual is engrossed in the task to enable escape from self-awareness of negative affect [14,32]. As noted above, the artificial nature of the task may have precluded the necessary level of engagement in order to fully evaluate the mood-repair function. Future research utilizing more naturalistic buying tasks could address this issue.

It may also be the case that different typologies of compulsive buyers exist and that those most susceptible 
to the mood repair function of buying behaviours were not included in the current sample, accounting for the null findings. Black [33] has suggested that subgroups of compulsive buyers may exist and may be motivated by different underlying diatheses that correspond to the varying diagnostic criteria that have been proposed for inclusion in DSM-V. Other researchers have also suggested that there may be a number of cognitive pathways to $\mathrm{CB}$ and therefore a range of $\mathrm{CB}$ manifestations ranging from compulsive to impulsive [34,35]. Clearly there is a need for a clinical measure of $\mathrm{CB}$ that draws on emerging research and captures the heterogeneity of $\mathrm{CB}$ behaviours and their consequences to allow for more refined investigations. In the absence of a current measure to allow for such sub-typing, the high rate of current or lifetime depression history $(73 \%)$ in the compulsive buying group provides the best assurance that the sample was representative of individuals proposed to be most susceptible to the mood repair function.

The results of the current study must be considered in light of a number of limitations. In addition to the artificial nature of the buying task, the experimental protocol did not include a comparison group in a "neutral" condition. This was partially due to the fact that the primary aim was to test the influence of negative mood rather than mood states per se, and the positive induction condition was included as a logical comparison condition to control for variation in baseline affect across participants. It is possible that demand characteristics influenced the post-induction affect ratings leading to an erroneous conclusion that the MIP was effective, but this seems unlikely given the extensive research that supports the validity of MIPs when taking into account participant demand (see [36] for a review). Given the high rate of mixed comorbidity reported in compulsive buyers [11] it may have been informative to compare responses to the buying task based on comorbid diagnosis (ICD, primary anxiety disorder, etc.). Although comorbid depression history was evaluated in this way, the relatively small sample sizes in the current study prohibited a more disorder-specific comparison, therefore future research may benefit from recruitment of larger samples specifically with this purpose in mind. Notwithstanding these limitations, the current study represents the first known experimental approach to test the mood repair hypothesis and to compare a group of clinically diagnosed compulsive buyers to pathological gamblers on measures of buying-relevant impulsivity. As such, the current study may provide a useful protocol that other researchers can modify or extend to further evaluate CB-relevant proposals.

In conclusion, the current findings did not support the mood repair hypothesis of compulsive buying. Additional research into this proposal as well as etiological factors is needed in order to provide a comprehensive model to account for these maladaptive behaviours and to help inform correct diagnostic classification if compulsive buying is to be considered to warrant inclusion in the DSM-V.

\section{ACKNOWLEDGEMENTS}

Funding for this study was provided by a National Health and Medical Research Council (NHMRC) of Australia Fellowship (630746) and a UNSW Faculty of Medicine Fellowship Enhancement Scheme awarded to A. D. Williams. The Author would like to thank Alicia Erskine and the St. Vincent's Hospital Gambling Treatment Program for assistance in recruitment and Aliza Werner-Seidler for assistance in data collection.

\section{REFERENCES}

[1] American Psychiatric Association (1994) Diagnostic and statistical manual of mental disorders. 4th Edition, American Psychiatric Association, Washington DC.

[2] McElroy, S., Keck, P.E., Pope, H.G., Smith, C.L. and Strakowski, S.M. (1994) Compulsive buying: A report of 20 cases. Journal of Clinical Psychiatry, 55, 242-248.

[3] Mueller, A., Mitchell, J.E., Crosby, R.D., Gefeller, O., Faber, R.J., Martin, A., et al. (2010) Estimated prevalence of compulsive buying in Germany and its association with sociodemographic characteristics and depressive symptoms. Psychiatry Research, 180, 137-142. doi:10.1016/j.psychres.2009.12.001

[4] Black, D.W. (1996) Compulsive buying: A review. Journal of Clinical Psychiatry, 57, 50-54.

[5] Lejoyeux, M., Bailly, F., Moula, H., Loi, S. and Adès, J. (1997) Study of compulsive buying in depressed patients. Journal of Clinical Psychiatry, 58, 169-173. doi:10.4088/JCP.v58n0406

[6] O'Guinn, T.C. and Faber, R.J. (1989) Compulsive buying: A phenomenological exploration. Journal of Consumer Research, 16, 147-157. doi:10.1086/209204

[7] Williams, A.D. (in press) Quality of life and psychiatric work impairment in compulsive buying: Increased symptom severity as a function of acquisition behaviours. Comprehensive Psychiatry.

[8] Christenson, G.A., Faber, J.R. and De Zwann, M. (1994) Compulsive buying: Descriptive characteristics and psychaitric comorbidity. Journal of Clinical Psychiatry, 55, 5-11.

[9] Schlosser, S., Black, D.W., Repertinger, S. and Freet, D. (1994) Compulsive buying. Demography, phenomenology, and comorbidity in 46 subjects. General Hospital Psychiatry, 16, 205-212. doi:10.1016/0163-8343(94)90103-1

[10] Miltenberger, R.G., Redlin, J., Crosby, R., Stickney, M., Mitchell, J., Wonderlich, S., et al. (2003) Direct and retrospective assessment of factors contributing to compulsive buying. Journal of Behavior Therapy and Experimental Psychiatry, 34, 1-9. 
doi:10.1016/S0005-7916(03)00002-8

[11] De Zwaan, M. (2011) Psychiatric comorbidity and compulsive buying compulsive buying: Clinical foundations and treatment. Routledge/Taylor and Francis Group, New York.

[12] Faber, R.J. and Christenson, G.A. (1996) In the mood to buy: Differences in the mood states experienced by compulsive buyers and other consumers. Psychology and Marketing, 13, 803-820. doi:10.1002/(SICI)1520-6793(199612)13:8<803::AID-M AR6>3.3.CO;2-2

[13] Williams, A.D. and Grisham, J. R. (in press) Impulsivity, emotion regulation, and mindful attentional focus in compulsive buying. Cognitive Therapy and Research.

[14] Kellett, S. and Bolton, J.V. (2009) Compulsive buying: A cognitive-behavioural model. Clinical Psychology \& Psychotherapy, 16, 83-99. doi:10.1002/cpp.585

[15] First, M.B., Spitzer, R.L., Gibbon, M. and Williams, J. (2002) Structured clinical interview for DSM-IV-TR Axis I disorders, research version. Biometrics Research, New York State Psychiatric Institute, New York.

[16] Watson, D., Clark, L.A. and Tellegen, A. (1988) Development and validation of brief measures of positive and negative affect: The PANAS scales. Journal of Personality and Social Psychology, 54, 1063-1070. doi:10.1037/0022-3514.54.6.1063

[17] Patton, J.H., Stanford, M.S. and Barratt, E.S. (1995) Factor structure of the barratt impulsiveness scale. Journal of Clinical Psychology, 51, 768-774. doi:10.1002/1097-4679(199511)51:6<768::AID-JCLP22 70510607>3.0.CO;2-1

[18] Stanford, M.S., Mathias, C.W., Dougherty, D.M., Lake, S.L., Anderson, N.E. and Patton, J.H. (2009) Fifty years of the Barratt Impulsiveness Scale: An update and review. Personality and Individual Differences, 47, 385-395. doi:10.1016/j.paid.2009.04.008

[19] Lovibond, S.H. and Lovibond, P.F. (1995) Manual for the depression anxiety and stress scales. 2nd Edition, Psychological Foundation, Sydney.

[20] Henry, J.D. and Crawford, J.R. (2005) The short-form version of the Depression Anxiety Stress Scales (DASS21): Construct validity and normative data in a large nonclinical sample. British Journal of Clinical Psychology, 44, 227-239. doi:10.1348/014466505X29657

[21] Monahan, P., Black, D.W. and Gabel, J. (1996) Reliability and validity of a scale to measure change in persons with compulsive buying. Psychiatry Research, 64, 59-67. doi:10.1016/0165-1781(96)02908-3

[22] Lesieur, H.R. and Blume, S.B. (1987) The South Oaks Gambling Screen (SOGS): A new instrument for the identification of pathological gamblers. American Journal of Psychiatry, 144, 1184-1188.

[23] Petry, N.M. (2003) Patterns and correlates of gamblers anonymous attendance in pathological seeking professional treatment. Addictive Behaviors, 28, 1049-1062.
doi:10.1016/S0306-4603(02)00233-2

[24] Stinchfield, R. (2002) Reliability, validity, and classification accuracy of the South Oaks Gambling Screen (SOGS). Addictive Behaviors, 27, 1-19. doi:10.1016/S0306-4603(00)00158-1

[25] First, M.B. (2008 draft) Structured clinical interview for DSM-IV-TR impulse control disorders not elsewhere classified (SCID-ICD). Biometrics Research Department.

[26] Velten, E. Jr. (1968) A laboratory task for induction of mood states. Behaviour Research and Therapy, 6, 473482. doi:10.1016/0005-7967(68)90028-4

[27] Gerrards-Hesse, A., Spies, K. and Hesse, F.W. (1994) Experimental inductions of emotional states and their effectiveness: A review. British Journal of Psychology, 85, 55-78. doi:10.1111/j.2044-8295.1994.tb02508.x

[28] Sinclair, R.C., Mark, M.M., Enzle, M.E., Borkovec, T.D., et al. (1994) Toward a multiple-method view of mood induction: The appropriateness of a modified Velten mood induction technique and the problems of procedures with group assignment to conditions. Basic and Applied Social Psychology, 15, 389-408. doi:10.1207/s15324834basp1504_1

[29] Koran, L.M., Chuong, H.W., Bullock, K.D. and Smith, S.C. (2003) Citalopram for compulsive shopping disorder: An open-label study followed by double-blind discontinuation. Journal of Clinical Psychiatry, 64, 793-798. doi:10.4088/JCP.v64n0709

[30] Black, D.W., Shaw, M. and Blum, N. (2010) Pathological gambling and compulsive buying: Do they fall within an obsessive-compulsive spectrum? Dialogues Clinical Neuroscience, 12, 175-185.

[31] Faber, R.J. (2011) Diagnosis and epidemiology of compulsive buying compulsive buying: Clinical foundations and treatment. Routledge/Taylor and Francis Group, New York.

[32] Faber, R.J. and Vohs, K.D. (2011) Self-regulation and spending: Evidence from impulsive and compulsive buying handbook of self-regulation: Research, theory, and applications. 2nd Edition, Guilford Press, New York.

[33] Black, D.W. (2007) A review of compulsive buying disorder. World Psychiatry, 6, 14-18.

[34] DeSarbo, W.S. and Edwards, E.A. (1996) Typologies of compulsive buying behavior: A constrained clusterwise regression approach. Journal of Consumer Psychology, 5, 231-262. doi: $10.1207 / \mathrm{s} 15327663$ jcp0503 02

[35] Nataraajan, R. and Goff, B.G. (1992). Manifestations of compulsiveness in the consumer-marketplace domain. Psychology and Marketing, 9, 31-44. doi:10.1002/mar.4220090105

[36] Westermann, R., Spies, K., Stahl, G. and Hesse, F.W. (1996) Relative effectiveness and validity of mood induction procedures: A meta-analysis. European Journal of Social Psychology, 26, 557-580. doi:10.1002/(SICI)1099-0992(199607)26:4<557::AID-EJ $\underline{\mathrm{SP} 769>3.3 . \mathrm{CO} ; 2-\mathrm{W}}$ 